\title{
Maximización de la producción de derivados lácteos mediante la metodología de la optimización lineal en la empresa Planta Lechera El Mantaro S.A.
}

\section{Maximization of the production of dairy products through the methodology of linear optimization in the Dairy Plant Company EI Mantaro S.A.}

\author{
Fidel O. Arauco C. ${ }^{(1)}$ y Felipe Arauco M.(1) \\ (1) Facultad de Ingenieria de Sistemas, Universidad Nacional del Centro del Perú \\ Email: foarauco@uncp.edu.pe
}

\section{RESUMEN}

El presente estudio está orientado a resolver el nivel bajo del beneficio de la empresa por medio de la maximización de la producción de derivados lácteos, mediante la producción tradicional establecida, los beneficios quincenales suman S/. 3030.00 soles. Aplicando las técnicas de la optimización lineal y utilizando eficientemente los recursos disponibles de la empresa, se maximiza la producción de derivados lácteos, logrando el incremento de los beneficios a la suma de $S / .9300 .00$ soles.

En la implementación metodológica, se planteó un nuevo modelo de optimización de la producción, para producir ocho tipos de derivados lácteos, se formuló el modelo de optimización lineal en su versión de maximizar, para la solución del mismo se utilizó el programa de optimización Lingo V. 13.0; la solución contempla el beneficio máximo a lograr y las cantidades a producir de cada derivado lácteo.

Las pruebas estadística de las hipótesis se realizaron utilizando la distribución T Student, para el tamaño de muestra con 8 valores, el grado de libertad es 7, el nivel de significancia elegido es el $5 \%$, entonces el valor teórico es 1.89, comparando los valores calculados del valor de t, para la hipótesis general e hipótesis específicas se ubican a la izquierda; es decir, en la región de aceptación de las hipótesis alternativas; por tanto, se aceptan quedando validado el modelo de optimización propuesto.

En conclusión, se logró obtener el diferencial en la producción hasta en tres veces superior, comparado al modelo tradicional.

Palabras claves: Maximización de la producción derivado lácteo - Optimización lineal - Eficiencia de los recursos - Programa Lingo.

\section{ABSTRACT}

The present study is oriented to solve the low level of the benefit of the company by means of the maximization of the production of dairy products, through the established traditional production, the biweekly profits total S/ 3030.00 soles. Applying the linear optimization techniques, efficiently using the available resources of the company, maximizes the production of dairy products, achieving the increase of profits to the sum of S/. 9300.00 soles.

In the methodological implementation, a new model of optimization of the production was proposed, to produce eight types of dairy products, the linear optimization model was formulated in its version of maximize, for the solution of the same was used the optimization program Lingo V 13.0; the solution contemplates the maximum benefit to be achieved and the quantities to be produced of each dairy derivative. It was possible to obtain the differential in the production, showing next.

Statistical tests of the hypotheses were performed using the student $T$ distribution, for the sample size with 8 values, the degree of freedom is 7 , the chosen level of significance is $5 \%$, then the theoretical value is 1.89 , comparing the values calculated from the value of $t$, for the general hypothesis and specific hypotheses are located on the left, ie in the region of acceptance of the alternative hypotheses, therefore, the proposed optimization model is accepted.

In conclusion, it was possible to obtain the differential in production up to three times higher, compared to the traditional model.

Key words: Production maximization - Dairy products Linear optimization - Resource efficiency - Lingo program. 


\section{INTRODUCCIÓN}

El administrador del negocio desea maximizar su producción, la planta lechera, se dedica a la fabricación y comercialización de helado, manjar blanco, mantequilla, queso y yogurt. El problema principal, radica en que las cantidades producidas de cada uno de los derivados lácteos no es la óptima, se da de acuerdo a la experiencia del jefe de planta y según la disponibilidad de materia prima e insumos. Además, no se apoya en modelo alguno ni se determina las cantidades en forma técnica, se da de acuerdo a la experiencia y los beneficios obtenidos que no son los óptimos, más bien se obtiene beneficio que está por debajo del óptimo.

En cuanto al estado económico actual de la empresa, la falta de una adecuada política de administración contable no ha permitido la aplicación de un sistema de costos reales de producción, lo que induce a fijar precios de ventas de los productos sin criterio técnico, que supuestamente no satisface las necesidades de crecimiento y desarrollo de la empresa; esto agravó el problema tributario, que por negligencia en su determinación correcta, ha devenido en una situación de pérdida de imagen y seriedad de la empresa hacia su entorno. El problema radica en que no existe un costeo técnico para la obtención de cada uno de los productos, que en esta investigación más adelante se realizará.

En este contexto se plantean los problemas de investigación, como sigue:

\section{Problema general}

¿Cuál es la influencia de la maximización de la producción de derivados lácteos en el beneficio de la empresa?

\section{Problemas específicos}

- ¿Cuál es la influencia de la maximización de la producción de queso en el beneficio de la empresa?

- ¿Cuál es la influencia de la maximización de la producción de yogurt en el beneficio de la empresa?

Mientras que los objetivos se mencionan a continuación:

\section{Objetivo general}

Incrementar los beneficios de la empresa mediante la maximización de la producción de derivados lácteos.

\section{Objetivos específicos}

- Medir la influencia de la maximización de la producción de queso en el beneficio de la empresa.

- Medir la influencia de la maximización de la producción de yogurt en el beneficio de la empresa.

En consecuencia, las hipótesis se plantean como sigue:

\section{Hipótesis general}

La maximización de la producción de derivados lácteos incrementa los beneficios de la empresa.

\section{Hipótesis específicas}

- La maximización de la producción de queso mejora el beneficio para la empresa.

- La maximización de la producción de yogurt incrementa el beneficio para la empresa.

\section{MATERIALES Y MÉTODOS}

\section{Tipo de investigación}

El tipo de investigación es aplicada, consiste en la aplicación de los métodos de optimización de sistemas en la maximización de la producción, para mejorar las utilidades de la empresa.

\section{Nivel de la investigación}

El trabajo de investigación consta de tres etapas, siendo las siguientes:

En la primera etapa es descriptivo; describe el sistema productivo y la demanda de la empresa en estudio. Los estudios de tipo descriptivos buscan describir y explicar las propiedades importantes de cualquier fenómeno o producto (Danhke, 1989).

El diseño correspondiente es el descriptivo simple cuya estructura es la siguiente:

$$
\mathrm{M} \longrightarrow \mathrm{O}
$$

Considerando:

M: Significa la muestra, la unidad de análisis.

O : Significa la observación, la información de los datos obtenidos mediante la encuesta.

La segunda etapa, consiste en el diseño y desarrollo de los modelos matemáticos para la maximización (optimización) de la producción de la empresa concordante con la demanda por cada uno de los productos, en esta etapa se utiliza el software de optimización matemática.

La tercera etapa es inferencial y explicativo, en base a los resultados de la solución del modelo de maximización del beneficio.

\section{Población y muestra}

La población la conforman la totalidad de las empresas elaboradoras de productos lácteos, que según fuentes del Ministerio de Industria, en el ámbito del estudio son dieciocho.

Por las facilidades otorgadas para este estudio, además por la capacidad de planta instalada, la muestra es dirigida.

\subsection{Procesamiento y análisis de datos}

El procesamiento y análisis de datos se realizará utilizando los aplicativos estadísticos SPSS, Excel y el software lingo, para la solución de los modelos de optimización lineal. 


\section{RESULTADOS}

El programa de optimización queda de la siguiente manera:

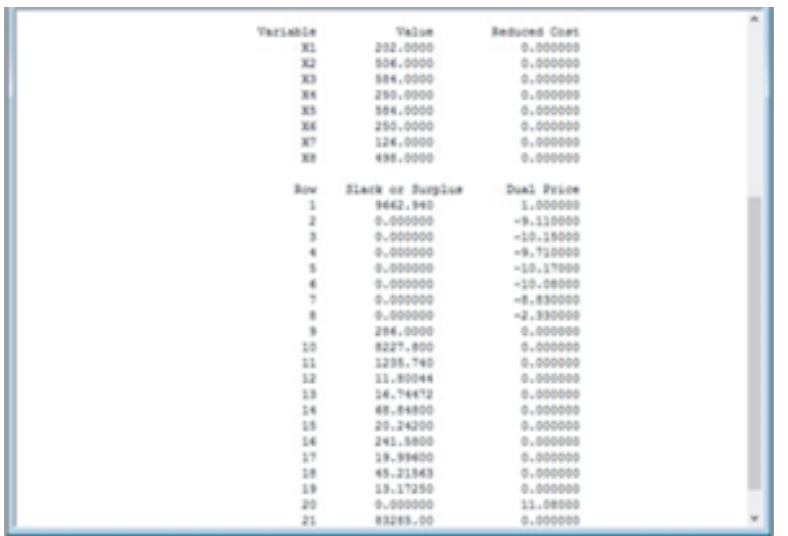

$\operatorname{Max}=1.97 * X 1+0.93 * X 2+1.37 * X 3+0.91 * X 4$ $+1.0 * \times 5+2$.

$25 * X 6+8.75 * X 7+11.08 * X 8$;

Demanda

Queso Fresco Semi-Integral:

$X 1>=202$;

Queso Fresco Light:

$X 2>=506$

Yogurt Batido Saborizado:

$X 3>=584$

$X 4>=250$

$X 5>=584$;

$X 6>=250$

$X 7>=126$

$X 8>=212$

Manjar Blanco:

Recursos

Leche (Litros):

$7 *(X 1+X 2)+0.9 *(X 3+X 4+X 5+X 6)+2.5 *(X 7)$

$<=15000$

Azúcar (Kilos):

$0.09(X 3+X 4+X 5+X 6)+0.43 X 8<=1600$

Sal (Kilos):

$0.025 X 7+0.00007(X 2+X 1) \quad<=15$

Cuajo (Kilos):

$0.01166 *(X 1+X 2) \quad<=25$

Calcio y Cloro (Kilos):

$0.044(X 2+X 1)<=100$

Saborizante (Kilos):

$0.006(X 5+X 4+X 3)+0.005 X 6<=30$

Mermelada (Kilos):

$0.13(X 5+X 6)<=350$

Sorbato de K (Kilos):

$0.003(X 5+X 4+X 3+X 6) \quad<=25$

Glucosa (Kilos):

$0.0296875 \times 8<=60$

Bicarbonato de $\mathrm{Na}$ (Kilos):

$0.02375 \times 8<=25$

Etiquetas: $X 1+X 2+X 3+X 4+X 5+X 6+X 7+X 8$ $<=3000$;
Presupuesto quincenal

$$
12.03 * \times 1+\quad 12.07 * X 2+
$$

$.13 * X 3+4.09 * X 4+3.13 * X 5+4.09 * X 6+$ $6.25 * X 7+6.91 * X 8<=100000.00$

Calcio y Cloro (Kilos): $0.044(X 2+X 1)<=100$

Saborizante (Kilos):

$0.006(X 5+X 4+X 3)+0.005 X 6<=30$

Mermelada (Kilos):

$0.13(X 5+X 6)<=350$

Sorbato de K (Kilos):

$0.003(X 5+X 4+X 3+X 6) \quad<=25$

Glucosa (Kilos):

$0.0296875 \times 8<=60$

Bicarbonato de $\mathrm{Na}$ (Kilos):

$0.02375 \times 8<=25$

Etiquetas:

$X 1+X 2+X 3+X 4+X 5+X 6+X 7+X 8<=3000$

Presupuesto quincenal

$$
\begin{aligned}
& 12.03 * X 1+\quad 12.07 * X 2+ \\
& .13 * X 3+4.09 * X 4+3.13 * X 5+4.09 * X 6+6.25 * \\
& X 7+6.91 * X 8<=100000.00 ;
\end{aligned}
$$

Solución del modelo matemático de optimización Para resolver el modelo matemático de optimización se

\begin{tabular}{|c|c|c|}
\hline 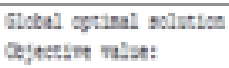 & & 966.9ง: \\
\hline Steariatistien: & & 8.000000 \\
\hline tocel salvez iterations & & . \\
\hline Zlegted :antat stoseds & & Q.:L \\
\hline Sodtl Chess! & & to \\
\hline toud retiakien: & $t$ & \\
\hline Scolinear vartwbleas & $\theta$ & \\
\hline Dest;e: 7a:tas:ts: & 8 & \\
\hline texal oongtzatoten & 22 & \\
\hline Sealisen: castraiers: & 8 & \\
\hline total sontestat & 61 & \\
\hline Jealiset: sgastesa: & : & \\
\hline
\end{tabular}
hizo uso del software aplicado LINGO.V13.0, previamente se editó el modelo en la interface de edición del programa, como se muestra al detalle:

Fuente: Solución del modelo con Lingo.

\section{DISCUSIÓN}

Antes de la implementación del modelo de optimización, la función objetivo y los beneficios por quincena de producción: S/.3 090.00. Luego de solucionar el modelo de optimización lineal propuesto, la función objetivo y los beneficios por quincena que se obtendrían suman S/.9663.00.

Las cantidades de derivados lácteos a producir por quincena se detallan a continuación: 
La producción de queso fresco semi integral $X 1$ es 202 $\mathrm{Kg}$., queso fresco $X 2506 \mathrm{Kg}$, yogurt batido saborizado X3 584 Lt., yogurt aflanado saborizado X4 250 Lt., yogurt batido frutado $X 5584 \mathrm{Lt}$., yogurt aflanado frutado X6 $250 \mathrm{Lt}$., mantequilla X7 $126 \mathrm{Kg}$. y manjar blanco X8 $498 \mathrm{Kg}$.

La solución del modelo propuesto para maximizar la producción, utilizando el programa Lingo, indica las cantidades que la empresa debe producir y los recursos que debe utilizar; solo así, se logrará la optimización de los beneficios. Optimizar significa encontrar el máximo beneficio; si se produce otras cantidades diferentes (puede ser más o menos cantidad) a lo que indica Lingo, el beneficio disminuirá y no será el óptimo.

\section{Prueba de la hipótesis general}

Planteando la hipótesis alternativa y la hipótesis nula de la siguiente manera:

- H1: La maximización de la producción de derivados lácteos incrementa el beneficio para la empresa.
- Ho: La maximización de la producción de derivados lácteos no incrementa el beneficio para la empresa.

Esta hipótesis se demostrará con dos grupos de datos, los correspondientes a la producción y ventas de la empresa actualmente como ocurre y el otro grupo la indicada por el programa lingo.

A continuación se realiza la prueba $T$ Student, mediante el programa SPSS se obtuvieron los resultados, que a continuación se muestran:

\section{Prueba $T$}

Tabla $\mathbf{N}^{\circ}$ 02. Estadisticas de muestras emparejadas

\begin{tabular}{|ccccc} 
& Media & N & $\begin{array}{c}\text { Desviación } \\
\text { estándar }\end{array}$ & $\begin{array}{c}\text { Media de } \\
\text { error estándar }\end{array}$ \\
\hline Par 1 prodproput & 375,00 & 8 & 186,253 & 65,850 \\
prodactual & 788,38 & 8 & 1368,338 & 483,781
\end{tabular}

Tabla $\mathbf{N}^{\circ}$ 03. Correlaciones de nuestrase emparejadas

\begin{tabular}{|cccc|} 
& N & Correlación & Sig. \\
\hline Par 1 prodproput \& prodactual & 8 &,- 398 &, 328
\end{tabular}

Tabla $\mathbf{N}^{\circ}$ 04. Correlaciones de muestras emparejadas

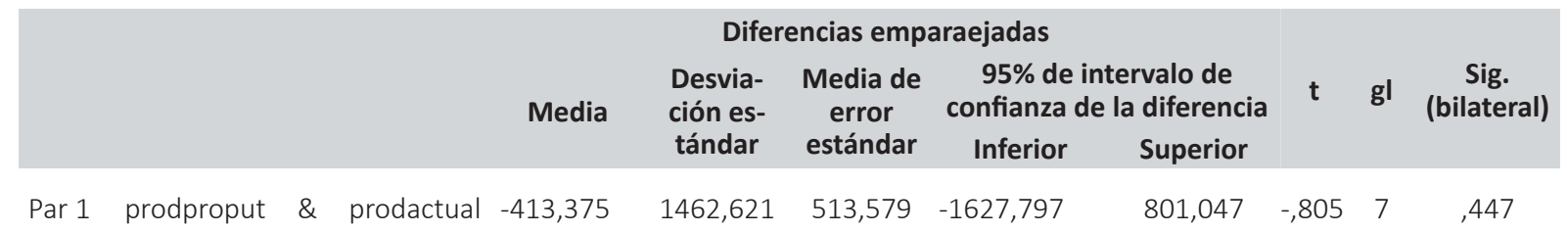

El valor calculado es 0.805 , se encuentra a la izquierda del valor teórico 1.89, se encuentra en la región de aceptación, por lo que se acepta la hipótesis alterna $H 1$ y se rechaza la hipótesis nula $H o$.

Con lo cual queda demostrada la hipótesis general, la maximización de la producción influye significativamente en las utilidades de la empresa.

\section{Prueba de la hipótesis específica 01}

Se formula la hipótesis alterna $H 1$ e hipótesis nula $H o$ de la siguiente manera:

- H1: La maximización de la producción de queso mejora el beneficio para la empresa.

- Ho: La maximización de la producción de queso no mejora el beneficio para la empresa.

Mediante el programa estadístico SPSS, para a prueba $T$ Student se obtuvo los siguientes resultados:

Tabla $\mathrm{N}^{\circ} 05$. Estadisticas de muestras relacionadas

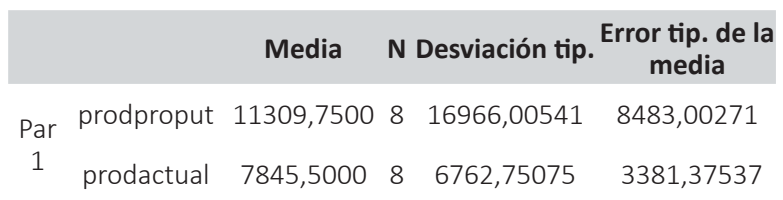

Tabla $\mathbf{N}^{\circ}$ 06. Correlaciones de muestras relacionadas

\begin{tabular}{cccc} 
& N $\begin{array}{c}\text { Desviación } \\
\text { tip. }\end{array}$ & $\begin{array}{c}\text { Error tip. de } \\
\text { la media }\end{array}$ \\
\hline Par UTIYOGU- UTILIDAD 1 & 4 &,- 300 &, 700 \\
\hline
\end{tabular}

Tabla $\mathbf{N}^{\circ}$ 07. Pruebas de muestras relacionadas

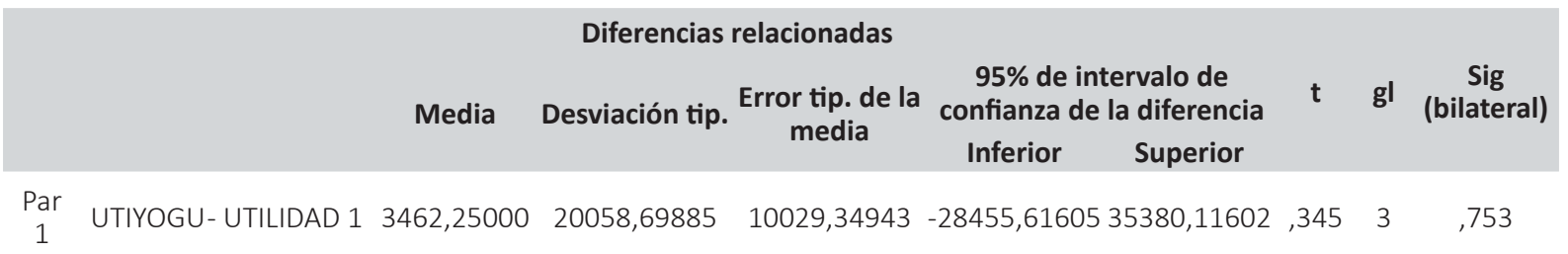


El valor calculado es 0.404 , se encuentra a la izquierda del valor teórico 1.89, en la región de aceptación, por lo que se acepta la hipótesis alterna $H 1$ y se rechaza la hipótesis nula $H o$.

Con lo cual queda demostrada esta hipótesis.

\section{Prueba de la hipótesis especifica 02}

Para demostrar esta hipótesis específica, se formula las hipótesis alterna $H 1$ e hipótesis nula $H_{o}$ :

- H1: La maximización de la producción de yogurt incrementa el beneficio para la empresa.

- Ho: La maximización de la producción de yogurt no incrementa el beneficio para la empresa.

Al aplicar la prueba $T$ Student, se obtuvieron los siguientes resultados:

El valor calculado es 0.345 , se encuentra a la izquierda del valor teórico 1.89, en la región de aceptación, por lo que se acepta la hipótesis alterna $H 1$ y se rechaza la hipótesis nula Ho.

Con lo cual queda demostrada esta hipótesis.

\section{CONCLUSIONES}

- La empresa al trabajar con su modelo de optimización lineal establecido, logra beneficios por $\mathrm{S} /$. 3090.00 nuevos soles quincenales, con cantidades limitadas de producción.

- En la investigación, se formuló el modelo de optimización lineal, considerando los 8 productos que la empresa comercializa, previamente se costeó y se encontró la cantidad de materiales y recursos necesarios por cada unidad de producto. Mediante el programa aplicado Lingo se obtuvo la solución óptima, logrando beneficios por la suma de $S / .9663 .00$ nuevos soles quincenalmente, lo que equivale a tres veces con respecto al modelo tradicional.

- Se ha demostrado la validez de las hipótesis planteadas en este estudio, que si la optimización de la producción de la empresa va a incrementar las utilidades de la misma. Todos los productos contribuyen a este objetivo; La prueba estadística utilizada fue la T Student con el apoyo del programa estadístico SPSS V. 20. Los valores calculados caen en la región de aceptación.

\section{REFERENCIAS BIBLIOGRÁFICAS}

Anderson et al. (1998). Métodos cuantitativos para los negocios. Thomson Editores. México.

Córdova, L. (2008). Investigación operativa: Modelo de programación lineal. Optimizar beneficios en la producción de derivados lácteos planta industrializadora de leche. Universidad de Córdova, Argentina.
Hillier, F. \& Lieberman, G. 2001. "Investigación de operaciones”. Séptima edición. Editorial Mc Graw-Hill. $1223 \mathrm{p}$

Talavera, S. (2002). Tesis de Maestría "Desarrollo de un modelo de programación lineal para el manejo de ecosistemas forestales”. Universidad Autónoma Nuevo León, México.

De La Peña, J. (1975). Tesis: Aplicación de la programación lineal en la industria de panificación. de: http://eprints.uanl.mx/7119/1/1080074564. PDF

Ojeda. R. (2011). Programación lineal para la asignación de personal a horarios de trabajo en una empresa de atención telefónica. http://congreso.investiga.fca.unam.mx/docs/xvii/ docs/D10.pdf

Tabo (2010). Empresa de C o f f e - W o o d International. https://docs.google.com/ viewer? ${ }_{\mathrm{v}} \&$ pid $={ }_{\text {sites }}{ }^{2} \quad$ srcid $=Z$ ZVVmYXVsdGRvbWFpbnxwcm9ncmFtY WNpb25saW5lYWwzOTh8Z3g6NjdlZDFjNGI2Yj Q3NWY5Mg.

Zevallos et al (2008). Optimización de ingresos en una empresa de productos lácteos Universidad del Salta, Argentina. 